\title{
Auroral radio emission and absorption of medium frequency radio waves observed in Iceland
}

\author{
Yuka Sato ${ }^{1}$, Takayuki Ono ${ }^{1}$, Masahide Iizima ${ }^{1}$, Atsushi Kumamoto ${ }^{1}$, \\ Natsuo Sato ${ }^{2}$, Akira Kadokura ${ }^{2}$, and Hiroshi Miyaoka ${ }^{2}$ \\ ${ }^{1}$ Graduate School of Science, Tohoku University, Japan \\ ${ }^{2}$ National Institute of Polar Research, Japan
}

(Received June 3, 2007; Revised November 30, 2007; Accepted December 3, 2007; Online published March 3, 2008)

\begin{abstract}
In order to study the generation and propagation processes of MF auroral radio emissions (referred to as auroral roar and MF burst) in the polar ionosphere, an Auroral Radio Spectrograph (ARS) system was installed at Husafell station in Iceland (invariant latitude: $65.3^{\circ}$ ). Data analysis of man-made transmissions also provides useful information for the ionosphere study as well as an investigation of auroral radio emissions since the propagation character of MF radio waves changes depending on electron-neutral collisions in the bottomside ionosphere. Thus, ionospheric absorption is examined in comparison with the solar zenith angle and auroral phenomena. The results indicate that the ARS data can be used to detect ionization occurring at distant regions. In late 2006, the ARS detected one auroral roar and two MF bursts, which were identified as left-handed polarized waves. Results of data analysis, including other auroral data and particle spectra observed by the DMSP satellite, suggest that the MF bursts are generated by electrons with an average energy of several keV associated with auroral breakup. On the other hand, the auroral roar is generated as upper hybrid waves by relatively low-energy electrons over the observation site and propagates downward, being converted into L-O mode electromagnetic waves.
\end{abstract}

Key words: Auroral roar, MF burst, absorption, propagation, polarization observation.

\section{Introduction}

Auroral roar and Medium-Frequency burst (MF burst) are members of auroral hectometric emissions, which have been detected at the ground-level. Some satellite observations resembling auroral roar have also been reported (James et al., 1974; Oya et al., 1985; Benson and Wong, 1987; Bale, 1999). Though radiation energy of the waveparticle interaction for these radio emissions is relatively small in comparison with Auroral Kilometric Radiation (AKR), study of the auroral radio emissions in the MF range is important for understanding the complex feature of the auroral phenomena generated in the polar ionosphere, planetary plasma and plasma in the universe (Labelle and Treumann, 2002).

Since the frequencies of auroral roar emissions are believed to be near the second (Kellogg and Monson, 1979, 1984) and third (Weatherwax et al., 1993) harmonics of the electron gyrofrequencies in source regions, they are referred to as $2 f_{c e}$ and $3 f_{c e}$ roar. MF burst shows a broadband impulsive feature (Weatherwax et al., 1994) with a bandwidth of $1-2 \mathrm{MHz}$ in a frequency range of $0.8-4.5 \mathrm{MHz}$ (LaBelle et al., 2005). The polarization character of $2 f_{c e}$ roar and MF burst have been identified as left-handed polarized waves by Shepherd et al. (1997), implying that they should propagate in the L-O mode in the ionosphere.

Copyright (c) The Society of Geomagnetism and Earth, Planetary and Space Sciences (SGEPSS); The Seismological Society of Japan; The Volcanological Society of Japan; The Geodetic Society of Japan; The Japanese Society for Planetary Sciences; TERRAPUB
Shepherd et al. (1999) showed the latitudinal dependence of auroral roar emissions, and it was reported that the intense auroral roar tended to appear in the poleward region of electrojets. They also examined Fast Auroral Snapshot (FAST) satellite data and compared simultaneous measurements of $2 f_{c e}$ roar emissions and precipitating electrons. It was suggested that auroral roar emissions were generated by inverted-V electrons; however, one-to-one correspondence between the location of the auroral electrojet and the source region of the auroral roar remains uncertain.

In order to clarify the nature of the MF auroral radio emissions and to understand their generation mechanism by observation, a new observation system named Auroral Radio Spectrograph (ARS) was developed at Husafell station in Iceland (invariant latitude: $65.3^{\circ}$ ). The ARS is designed to identify the spectra and polarization character of radio waves in the MF range, especially covering $3 f_{c e}$ roar, whose polarization character has not yet been clarified.

After being refurbished in late 2006, the ARS was able to detect the auroral roar and MF burst events successfully. The ARS observation data are analyzed in comparison with other data, such as geomagnetic field, riometer and auroral image data recorded at the ground-level and also auroral particle data observed by the SSJ/4 onboard the DMSP (Defense Meteorological Satellite Program) satellites F13, F15, and F16. Within the data analysis of the present dataset, the DMSP satellites follow no conjugate path over the Husafell station simultaneously with the auroral roar and MF burst events detected by the ARS. However, characteristics of au- 


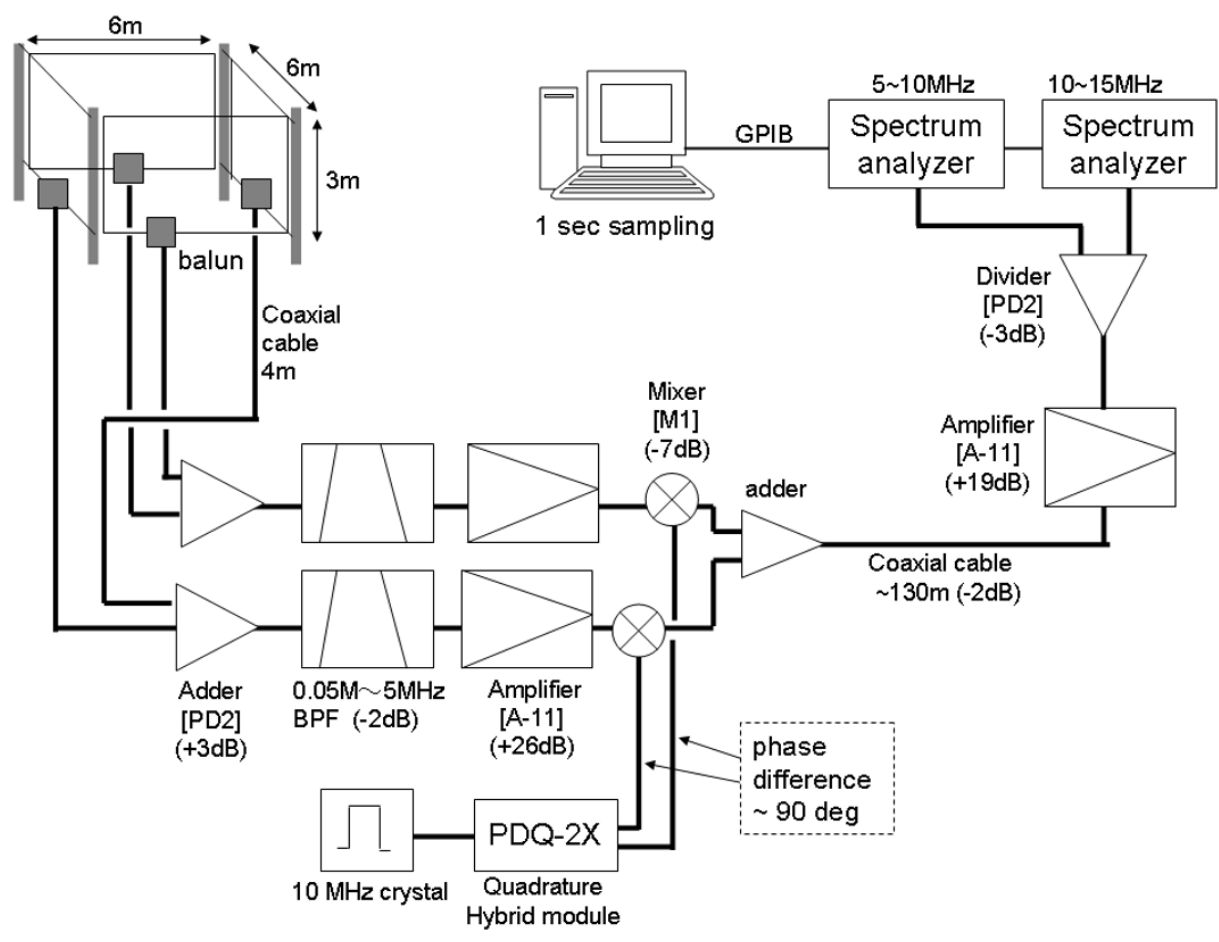

Fig. 1. A system block diagram of the ARS installed at the Husafell station in Iceland.

roral electrons precipitating near the station and position of auroral arc can be inferred by comparison with the DMSP data obtained in the conjugate region of the southern hemisphere.

To clarify the frequency spectra and occurrence character of the auroral radio emissions, it is necessary to take into account the nature of radio wave absorption in the ionosphere, as pointed out by Weatherwax et al. (1995). This is because occurrence of the auroral radio emissions observed on the ground is certainly controlled by the collisional damping effect in the $\mathrm{D}$ and $\mathrm{E}$ regions of the ionosphere. It is noted that auroral absorption phenomena involve information of the auroral ionization and the magnetosphere-ionosphere interaction as well as generation of the auroral radio emissions in the ionosphere. Therefore, in this paper, the nature of ionospheric absorption is also examined, including auroral absorption with a short time scale.

\section{Instrumentation}

The ARS was set in September 2005 for observation of auroral radio emission in the MF range and was further improved in September 2006. As shown in Fig. 1, the frontend block of the ARS consists of loop antennas, baluns, electrical circuits of band pass filters, amplifiers and a polarization separator. The back-end block consists of an amplifier, two spectrum analyzers, a personal computer (PC), and a GPS receiver.

To detect the weak auroral radio waves on the ground, the ARS antenna was designed with four magnetic loop antenna elements, each with a size of $2.7 \times 6.0 \mathrm{~m}$. Two parallel pairs of loops are set up in the north-south and east-west direction, respectively. The band pass filter is a passive type filter made of capacitors and inductors and consists of an 11th order low-pass filter and a 6th order high-pass filter whose cutoff frequencies are $5 \mathrm{MHz}$ and $0.05 \mathrm{MHz}$, respectively. The polarization separator consists of two mixers, an adder and a local signal generator. The local signals with a phase difference of $90^{\circ}$ are generated by a $10-\mathrm{MHz}$ crystal oscillator and a quadrature hybrid module.

The present instrument uses a unique technique to separate the left-handed and right-handed circularly polarized components of coherent signals detected by the orthogonal antennas of X (north-south direction) and Y (east-west direction). The left-handed circular component (LH) picked up from the $\mathrm{X}$ and $\mathrm{Y}$ antennas are described as:

$$
\begin{aligned}
& \mathrm{X}_{\mathrm{LH}}=a_{\mathrm{LH}} \cos \omega t \\
& \mathrm{Y}_{\mathrm{LH}}=a_{\mathrm{LH}} \sin \omega t
\end{aligned}
$$

where $a_{\mathrm{LH}}$ and $\omega$ are the amplitude and the frequency of the left-handed polarized signal, respectively.

By mixing them with the local signals of $\mathrm{LO}_{\mathrm{X}}=\cos \omega_{0} t$ and $\mathrm{LO}_{\mathrm{Y}}=\sin \omega_{0} t$, which have phase difference of $90^{\circ}$ as shown in Fig. 1, the following equations are obtained:

$$
\mathrm{X}_{\mathrm{LH}} \times \mathrm{LO}_{\mathrm{X}}=\frac{a_{\mathrm{LH}}}{2}\left[\cos \left(\omega+\omega_{0}\right) t+\cos \left(\omega-\omega_{0}\right) t\right]
$$

and

$$
\mathrm{Y}_{\mathrm{LH}} \times \mathrm{LO}_{\mathrm{Y}}=\frac{a_{\mathrm{LH}}}{2}\left[-\cos \left(\omega+\omega_{0}\right) t+\cos \left(\omega-\omega_{0}\right) t\right]
$$

Adding these signals, the amplitude of the left-handed polarized signal appearing at the frequency of $\left|\omega-\omega_{0}\right|$ is as follows:

$$
\begin{aligned}
\operatorname{Output}(\mathrm{LH}) & =\left(\mathrm{X}_{\mathrm{LH}} \times \mathrm{LO}_{\mathrm{X}}+\mathrm{Y}_{\mathrm{LH}} \times \mathrm{LO}_{\mathrm{Y}}\right) \\
& =a_{\mathrm{LH}} \cos \left(\omega-\omega_{0}\right) t
\end{aligned}
$$




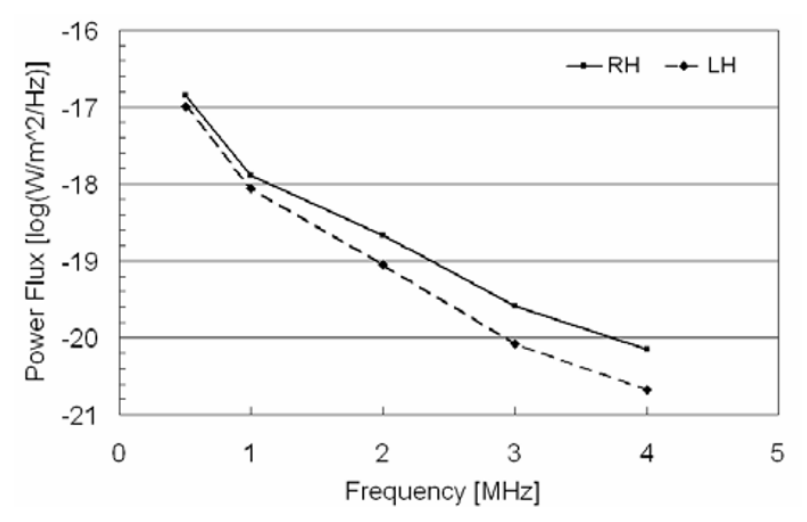

Fig. 2. The frequency dependence of system noise level plotted as the equivalent power flux of electromagnetic waves. The solid and dashed lines represent RH and LH polarized wave components, respectively.

On the other hand, the right-handed circular component (RH) picked up from the $\mathrm{X}$ and $\mathrm{Y}$ antennas are described as follows:

$$
\begin{aligned}
& \mathrm{X}_{\mathrm{RH}}=a_{\mathrm{RH}} \cos \omega t \\
& \mathrm{Y}_{\mathrm{RH}}=-a_{\mathrm{RH}} \sin \omega t
\end{aligned}
$$

In the same way, the amplitude of the right-handed polarized signal $a_{\mathrm{RH}}$ is obtained by the following equation:

$$
\operatorname{Output}(\mathrm{RH})=a_{\mathrm{RH}} \cos \left(\omega+\omega_{0}\right) t
$$

Equations (4) and (6) show that the amplitude of the LH component is obtained in the frequency band lower than the local signal, while the RH component is obtained in the frequency band higher than it. For the present instrumentation, $\omega_{0}$ was set to $10 \mathrm{MHz}$; thus, the output signals with the frequency of $8 \mathrm{MHz}$ and $12 \mathrm{MHz}$ correspond to the power of the $\mathrm{LH}$ and $\mathrm{RH}$ polarized waves for the observation frequency of $2 \mathrm{MHz}$, respectively. This method has an advantage in that it needs only one signal transmission line from the back-end block in the observation room to the front-end of the main receiver set up near the loop antenna site as shown in Fig. 1. It is noted that for the display of observation results of $\mathrm{RH}$ and $\mathrm{LH}$ polarization components, frequency is converted from $10-15 \mathrm{MHz}$ to $0-5 \mathrm{MHz}$ for the $\mathrm{RH}$ polarization and from $5-10 \mathrm{MHz}$ to $0-5 \mathrm{MHz}$ for the LH polarization. However, due to frequency response of the low-pass and high-pass filters, the frequency coverage of the ARS receiver is identified as $0.05-5.0 \mathrm{MHz}$.

The front-end and back-end blocks are connected by a coaxial cable by which signals as well as electrical power are transmitted. The observed signals are finally input into the spectrum analyzers, and the PC controls the spectrum analyzers via the General Purpose Interface Bus (GPIB). The PC acquires the observation time and other observation parameters of the spectrum analyzers every $1 \mathrm{~s}$, as well as data with the frequency range from 5 to $15 \mathrm{MHz}$. The ARS is designed to be operated continuously except when the DVD-RAM media is exchanged by the operator every 20 days.

In order to evaluate the absolute sensitivity of the ARS, calibration was carried by MF radio waves transmitted from a standard loop antenna. Figure 2 shows the equivalent power flux for the background system noise level of the receiver, comparing a typical intensity range of $\mathrm{MF}$ auroral radio waves from $6.6 \times 10^{-18}-26.6 \times 10^{-18} \mathrm{~W} / \mathrm{m}^{2} / \mathrm{Hz}$ reported by LaBelle and Treumann (2002). As shown in Fig. 2, the ARS has enough sensitivity for detecting MF auroral radio emissions with typical intensity.

\section{Observation Data}

\subsection{Ionospheric absorption of man-made MF radio waves}

Data from the ARS observation include not only auroral radio waves but also numerous sferics and man-made radio waves. A large number of man-made MF radio waves create a cluster of signals from distant radio stations. Data analysis of these wide band signals provides useful information for a study of ionosphere as well as auroral radio emission. The propagation character of MF radio waves is variable depending on the ionospheric condition, especially the electron-neutral collision, which is controlled by the solar zenith angle and solar and geomagnetic activities. Thus, it is worth examining the collisional damping effect on long distance radio wave signals measured by the ARS. Ionospheric absorption increases with an increase in the electron number density of the low altitude region of the ionosphere as well as neutral gas density. In the polar ionosphere, the ionization is given by the solar EUV radiation and auroral particle precipitation. Thus, it is possible to analyze the ARS data for the investigation of ionospheric absorption depending on solar zenith angle and absorption due to the auroral ionization.

3.1.1 Solar zenith angle dependence Figure 3(a) shows an example of the daily dynamic spectrum within a frequency range of $0.8-5.0 \mathrm{MHz}$ observed on January 10,2006 , when the geomagnetic activity is classified as a quiet day $\left(\Sigma K_{p}=2+\right)$. All horizontal lines are regarded as man-made signals. On geomagnetically quiet days, distant man-made signals tend to fade out in association with the sunrise. As shown in Fig. 3(a), the received power of manmade transmissions in the lower frequency range tends to fade out earlier than that of the higher frequency signals. This tendency is consistent with the report by LaBelle et al. (1994). The intensity of the broadcast signals from distant stations tends to recover with the sunset. The recovery of the broadcast signals in the higher frequency range tends to be earlier than that of lower frequency signals.

In general, the magnitude of absorption in the lower ionosphere $(\Gamma)$ is described by the following equation:

$$
\Gamma[\mathrm{dB}]=4.6 \times 10^{-5} \int \frac{v n}{\left(\omega^{2}+v^{2}\right)} d l
$$

where $d l, \omega, v$, and $n$ are the element of propagation path, the radio wave frequency, the electron-neutral collisional frequency and the electron density, respectively (Kelley, 1989). Equation (7) shows that lower frequency radio waves are absorbed more severely than higher frequency waves. Intense signals below 1.6 MHz can be found in Fig. 3(a). In fact, the frequency band from 531 to $1602 \mathrm{kHz}$ is assigned for AM radio broadcasting signals. The averaged power within the frequency band from 0.8 to $1.6 \mathrm{MHz}$ 


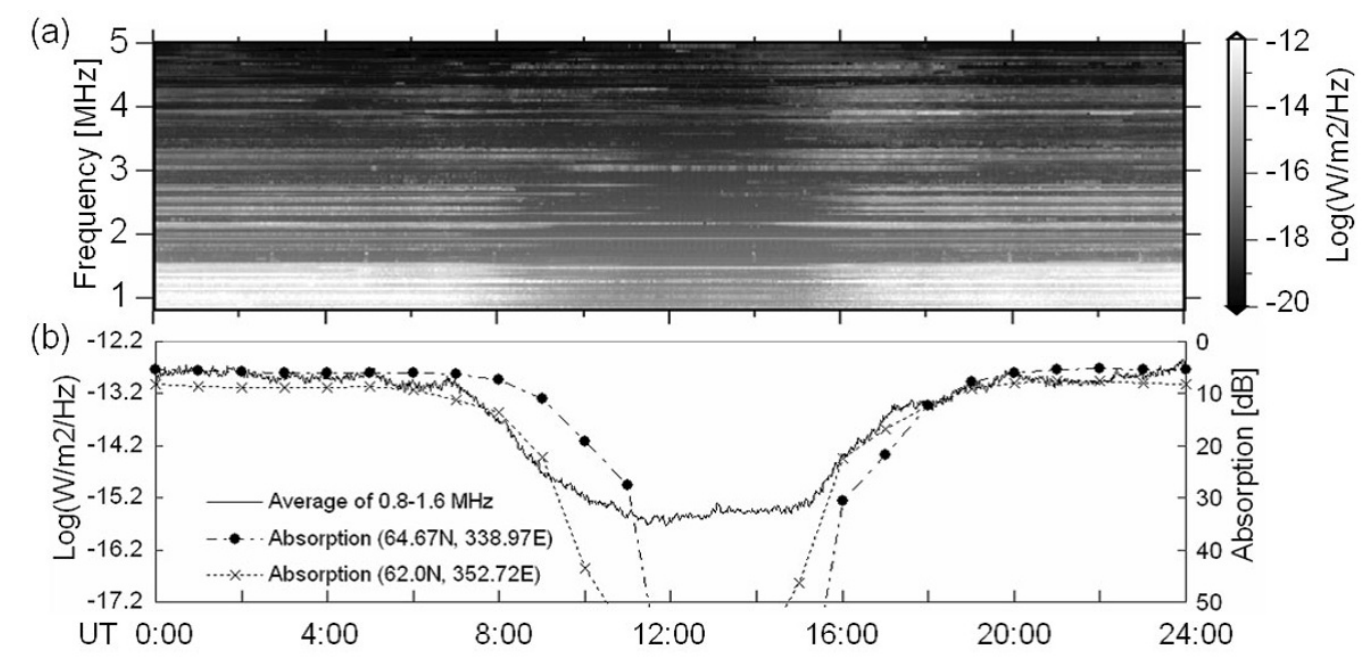

Fig. 3. (a) An example of the daily dynamic spectrum within a frequency range of 0.8-5.0 MHz observed on January 10, 2006. (b) The averaged power within the frequency band from 0.8 to $1.6 \mathrm{MHz}$ (the solid line). The dashed line with dots $(\bullet)$ and the doted line with crosses $(\times)$ show calculated trends of absorption for the one hop propagation model. The lines with dots and crosses correspond to the results by assuming reflection points above the Husafell station $(64.67 \mathrm{~N}, 339 \mathrm{E})$ and at the point of $(62.0 \mathrm{E}, 353 \mathrm{E})$, respectively.

is plotted by a solid curve in Fig. 3(b). Because the temporal variation of broadcasting signal intensity is controlled by the ionospheric absorption effect, data analysis of this absorption phenomenon reveals a general feature of ionospheric absorption due to ionization of low-altitude ionosphere. So, the observed variation is compared with calculated absorption $\Gamma$ expressed by Eq. (7) by assuming a simple one-hop propagation path. In this calculation, the MSIS and IRI models are used to obtain values of $v$ and $n$, respectively. First, it is assumed that the absorption is controlled by the ionization above the Husafell station, i.e. the reflection point is located above the station. The temporal variation of the absorption magnitude from this model, shown by a dashed line with dots (•) in Fig. 3(b), does not show the real temporal change which is shown by the solid line in the same figure. There is a time lag of $55 \mathrm{~min}$. This time lag suggests that the observed absorption may be controlled by the ionization in a different local time region. Next, the best fit propagation path with a distant reflection point is examined. Considering the time lag of $55 \mathrm{~min}$, the absorption point (i.e. reflection point at a low-altitude ionosphere) should be located along 353E meridian (see Fig. 4). Considering the secant law, the best-fit altitude of reflection point and incident angle are roughly estimated at about $85 \mathrm{~km}$ and $80^{\circ}$, respectively. The calculated absorption $\Gamma$ is shown by a dotted curve with crosses $(\times)$ in Fig. 3(b). The best fit condition leads the reflection point at $353 \mathrm{E}$ longitude and $62 \mathrm{~N}$ latitude.

3.1.2 Auroral activity dependence (auroral absorption) Figure 5(a) shows a dynamic spectrum in the frequency range of $0-5 \mathrm{MHz}$ obtained for $18 \mathrm{~h}$ beginning at 1500 UT on September 23, 2006. Figure 5(b) displays variations of averaged powers in the frequency ranges of 1.41.6 MHz and 4.1-4.4 MHz. Figure 5(c) and 5(d) shows the $\mathrm{H}$ component of the magnetometer data and riometer data recorded at Tjornes station in Iceland, respectively. The riometer data provide temporal variation of cosmic noise in- tensity at $30 \mathrm{MHz}$ to obtain cosmic noise absorption (CNA) associated with the energetic particle precipitation. Because geomagnetic data and riometer data were not available at the Husafell station at that time, we used the data obtained at the Tjornes station, which is located in the northeast part of Iceland (invariant latitude of $66.38^{\circ}$ and magnetic longitude of $71.08^{\circ}$ ) as shown in Fig. 4.

After the end of the normal absorption effect due to the sunset, broadcasting signals appeared in a wide frequency range around $1800 \mathrm{UT}$. At $1840 \mathrm{UT}$, an abrupt decrease in the signal intensity appeared. At 2105 UT, the most significant absorption occurred and lasted for more than $30 \mathrm{~min}$. In this event, absorption took place throughout the frequency range of the receiver from 0.5 to $5 \mathrm{MHz}$. As shown in Fig. 5(a), there were many absorption events appearing through the night. In general, absorption of the man-made signals simultaneously occurs in both LH- and RH-polarized components. Night time absorption events were associated with geomagnetic auroral and CNA activities. When we compare the magnitude of MF radio wave absorption with CNA data, it was measured as almost $20 \mathrm{~dB}$ while the CNA data varied by only $2 \mathrm{~dB}$. The MF radio absorption event started within about 20 min prior to the auroral breakup. On the other hand, the abrupt absorption in the higher frequency range $(4.1-4.4 \mathrm{MHz})$ occurred at 1840 UT with a clear time lag from the onset of geomagnetic and CNA disturbances measured at the Tjornes station. An absorption event occurred at 1840 UT and was delayed by $20 \mathrm{~min}$ after the onset of the CNA event associated with a small fluctuation signature within positive bay event of the $\mathrm{H}$ component geomagnetic field. These time lags can be interpreted as spatial differences of ionospheric regions monitored by these observation instruments; namely, sometimes auroral absorption detected by the ARS did not show a one-to-one correspondence with other auroral activities observed at the station.

In order to investigate rapid behavior of auroral absorp- 


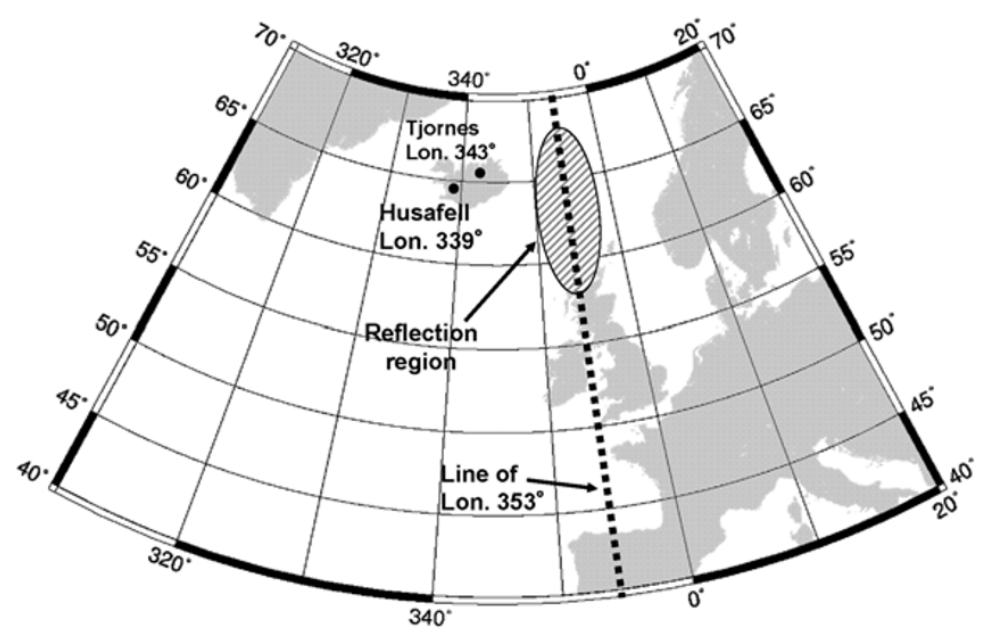

Fig. 4. A geographic map near Iceland. The dashed line shows the longitude of 353E, and the shaded area is estimated 'reflection region' where most of distant man-made transmissions observed by the ARS are absorbed and reflected.

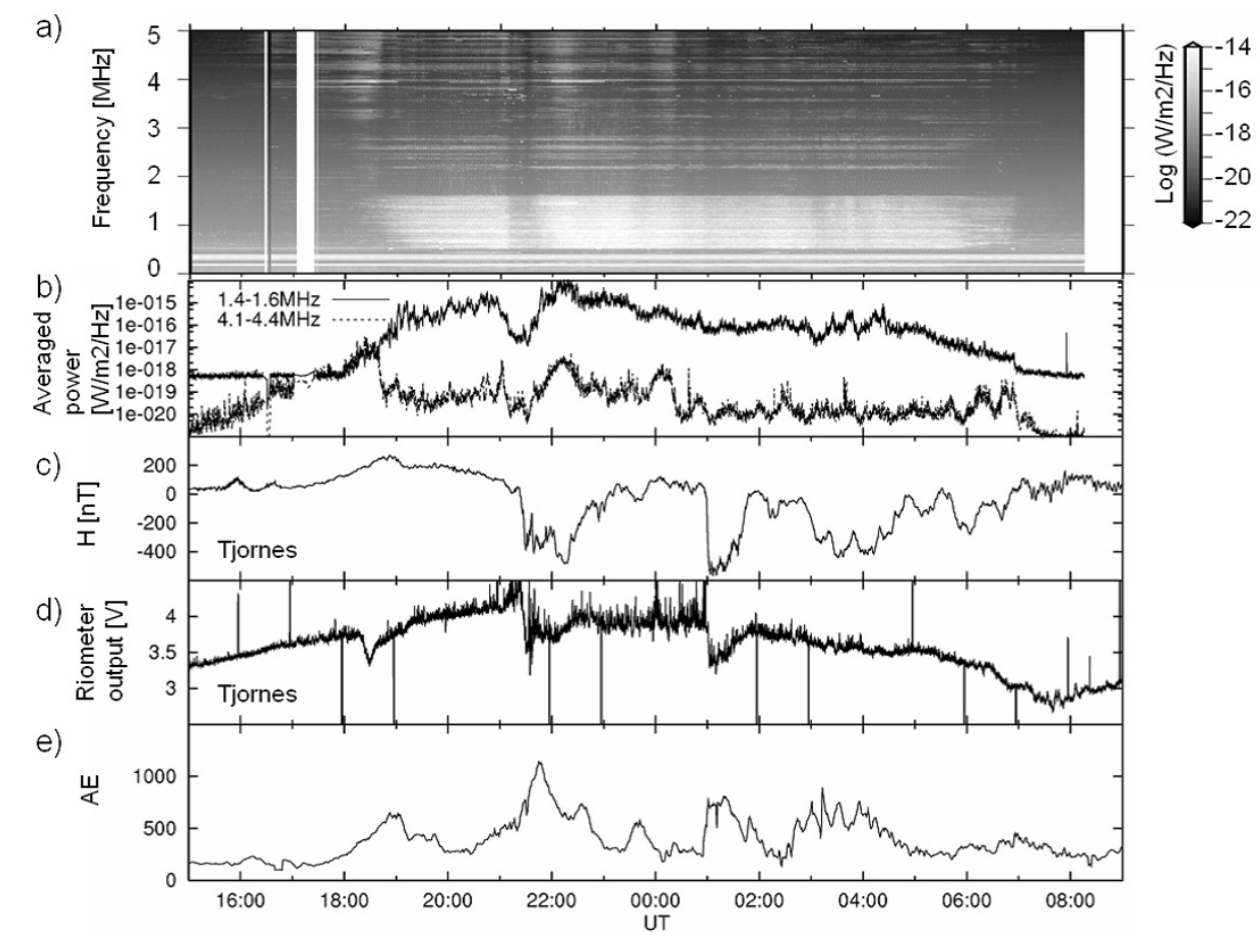

Fig. 5. Auroral absorption event associated with auroral activities from 1500 UT on September 23 to 0900 UT on September 24, 2006. There are plots of (a) a dynamic spectrum in the frequency range of $0-5 \mathrm{MHz}$, (b) variations of averaged powers for frequency ranges of 1.4-1.6 $\mathrm{MHz}$ and $4.1-4.4$ $\mathrm{MHz}$, (c) the $\mathrm{H}$ component of the geomagnetic field data at the Tjornes station, (d) riometer data at the Tjornes station, and (e) AE index.

tion, we examined ARS data in comparison with auroral pulsation in the Pc4 range obtained on September 25, 2006. Figure 6(a) and 6(b) shows a dynamic spectrum for a 0 $2 \mathrm{MHz}$ frequency range observed from 0430 to $0505 \mathrm{UT}$ and the variation of averaged power for a frequency range of $1.4-1.6 \mathrm{MHz}$, respectively. At this time, auroral pulsation appeared in early morning hours after auroral breakup, which occurred at 2236 UT on September 24.

Also, at this time, an auroral TV camera (ATV) operated through the night. As a result, we were able to examine the temporal variation of auroral absorption detected by the
ARS and compare it with the intensity variation of auroral emissions as well as ULF and CNA activities observed at the Tjornes and Syowa stations. In Fig. 6(c), relative auroral intensity averaged over the east and north part of the sky are represented by two curves of "aveE" and "aveN", showing that a pulsating arc was appearing with a periodicity of $111 \mathrm{~s}$. It is noted that the gradual increase of the curve of "aveE" data is due to the twilight, not the aurora. Figure $6(\mathrm{~d})$ and $6(\mathrm{e})$ shows the $\mathrm{H}$ component of the induction magnetometer data and the riometer data recorded at Syowa station in Antarctica, which is located at the geo- 


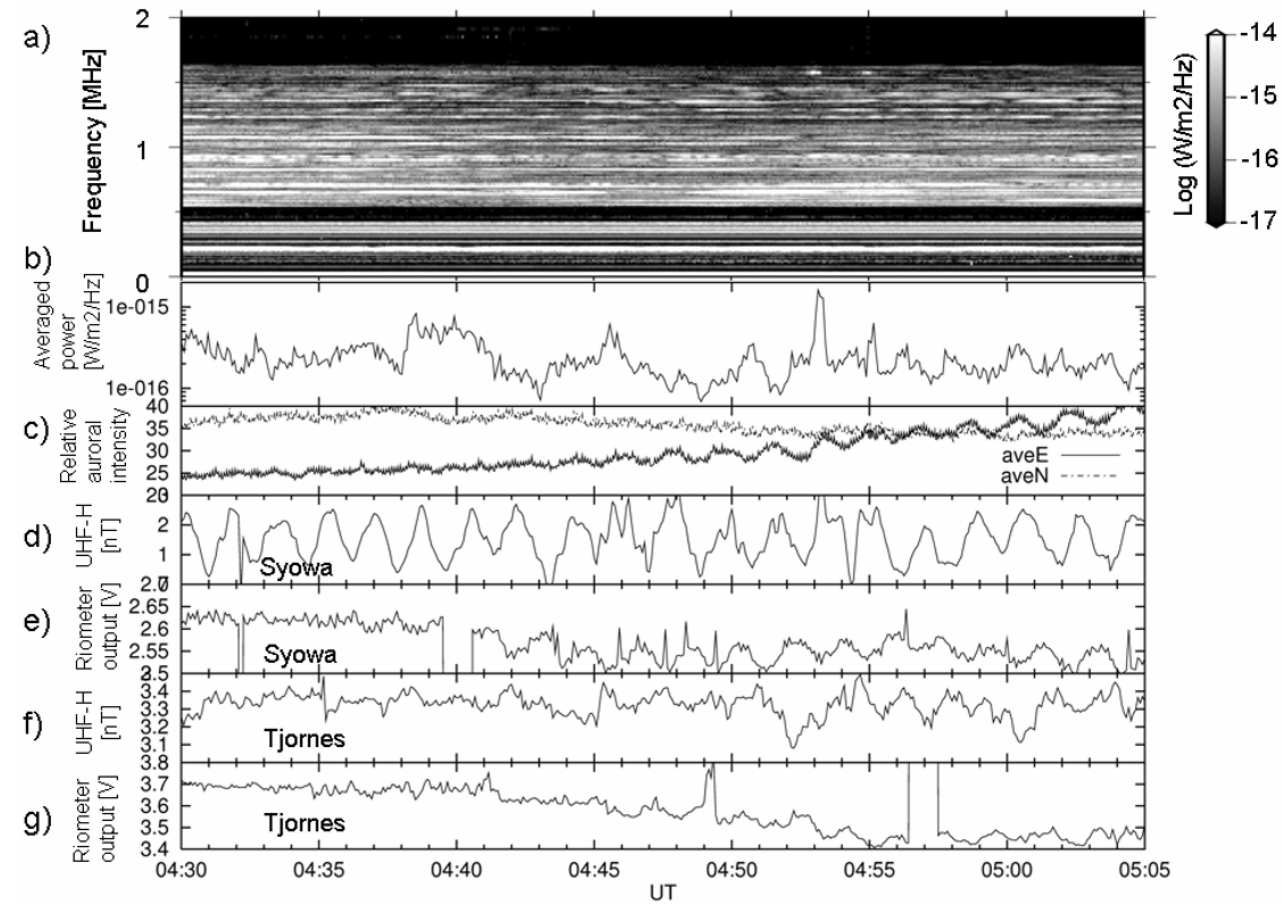

Fig. 6. Auroral absorption event associated with Pc4 range auroral pulsation observed between 0430 UT and 0505 UT on September 25, 2006 . There are plots of (a) a dynamic spectrum for the $0-2 \mathrm{MHz}$ frequency range, (b) variation of averaged power for a frequency range from 1.4 to $1.6 \mathrm{MHz}$, (c) relative auroral intensity averaged over the east and north part of the sky: "aveE" and "aveN", (d) the H component induction magnetometer at Syowa, (e) riometer data at Syowa, (f) the $\mathrm{H}$ component induction magnetometer at Tjornes, and (g) riometer data at Tjornes.

magnetic conjugate region of Husafell. Figure 6(f) and 6(g) shows the $\mathrm{H}$ component of the induction magnetometer data and the riometer data recorded at the Tjornes station. Although there are some data gaps in the geomagnetic data at the Tjornes station near 0449 UT and 0457 UT, we can clearly see conjugate behavior of ULF and CNA pulsations between the northern and southern hemisphere. Fourier analysis of the Syowa ULF data indicates that there is clear periodicity of $111 \mathrm{~s}$. The pulsation signature also appears in the ARS data after 0450 UT with the repetition period of $90 \mathrm{~s}$, as shown in Fig. 6(b). Although there is a small difference of periodicity, it is inferred that periodic absorption found in the MF radio wave signal may be caused by periodic ionization due to pulsating auroras.

\subsection{Auroral roar (November 10, 2006)}

Within the observation period from September to November, 2006, one auroral roar event and two MF burst events were detected. Figure 7 shows the auroral roar event obtained around 0118 UT on November 10, 2006. Figure 7(a)-(c) is the Auroral Electrojet (AE) index, the $\mathrm{H}$ component of the geomagnetic field at the Husafell station, and the riometer data at the Tjornes station from 1500 UT on November 9 to 0900 UT on November 10, 2006, respectively. Figure 7(d) shows a dynamic spectrum of ARS data for the same time period. Auroral absorption began near 1900 UT associated with the onset of a positive bay of the $\mathrm{H}$ component magnetic field observed at the Husafell station, as shown in Fig. 7(b). The auroral activity continued as a relatively disturbed feature through the night with a maximum excursion of $\mathrm{H}$ component of the magnetic field of $-457 \mathrm{nT}$. The time period between the two dashed lines in Fig. 7 includes the appearance of the auroral roar. It is noted that, during the auroral roar event, there was no significant change in the magnetic field at the Husafell station. Figure 7(e) and 7(f) shows the left-handed (LH) and right-handed $(\mathrm{RH})$ polarization components of the ARS data recorded during the auroral roar event from 0113 UT to 0123 UT. The spectrum of the auroral roar is intensified at $0115 \mathrm{UT}$ lasting for $4.5 \mathrm{~min}$. The central frequency varies between 3.82 and $3.87 \mathrm{MHz}$ and the frequency bandwidth is within a range of $50-130 \mathrm{kHz}$. The peak intensity was measured as about $3 \times 10^{-20}-10^{-19} \mathrm{~W} / \mathrm{m}^{2} / \mathrm{Hz}$. Considering the cyclotron frequency near the ionosphere, the present auroral roar emission can be recognized as a $3 f_{c e}$ roar emission. The intensity ratio of the $\mathrm{LH}$ and $\mathrm{RH}$ waves was measured as more than $10 \mathrm{~dB}$. The observed $3 f_{c e}$ roar was then identified as a left-handed polarized wave.

3.3 MF burst (September 23 and October 28, 2006)

Two MF burst events were detected by the ARS on September 23 and October 28, 2006. Figure 5(a) in Subsection 3.1 includes the MF burst event on September 23. The dashed line in Fig. 5 indicates the time when the MF burst occurred in association with auroral breakup with the maximum AE of about $1300 \mathrm{nT}$ recorded near 2123 UT. Figure 8(a) and 8(b) shows dynamic spectra of LH and RH components of ARS data recorded at 2121-2125 UT. The MF burst can be identified as broadband emissions covering from 1.9 to $2.6 \mathrm{MHz}$ appearing only in the LH spectra. The polarization character of this MF burst event is consistent with the result reported by Shepherd et al. (1997). However, it is noted that the present event lasts for only a short time period (10 s), while the typical duration has been reported to 


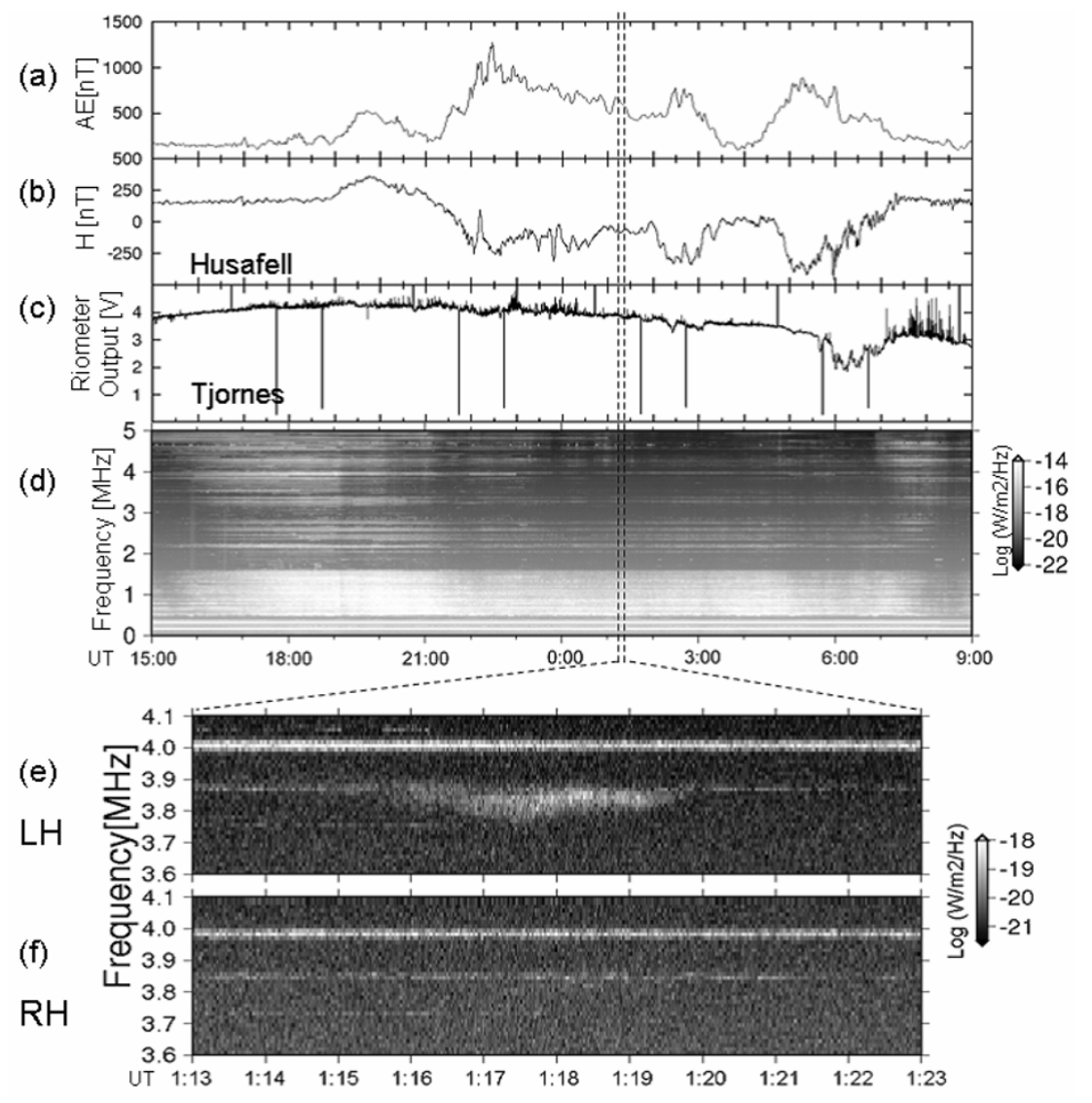

Fig. 7. The auroral roar event obtained around 0118 UT on November 10, 2006. The upper panels show (a) the AE index, (b) the H component of the geomagnetic field at Husafell, (c) riometer data at Tjornes and (d) a dynamic spectrum of ARS data observed from 1500 UT on November 9 to 0900 UT on November 10, 2006. The lower panels (e) and (f) are expanded dynamic spectra showing left-handed (LH) and right-handed (RH) polarization components of ARS data obtained 0113-0123 UT on November 10, 2006.

occur for about several minutes (Weatherwax et al., 1995; LaBelle et al., 1997). Figure 8(c) shows a series of auroral images of $427.8 \mathrm{~nm}$ recorded by the Conjugate Auroral Imager (CAI) instrument, which was located in the Husafell station. When the MF burst appeared at 2123:10 UT, the auroral activity was not so strong, and it was located in the southern part of the sky. The auroral breakup started at 2125:00 UT, and it was delayed by more than $1 \mathrm{~min}$ after the MF burst event.

Figure 9 shows the second MF burst event obtained from 1500 UT on November 9 to 0900 UT on November 10, 2006. Figure 9(a)-(d) shows the AE index, the H component of the geomagnetic field at the Husafell station, riometer data at the Tjornes station and the dynamic spectrum of the ARS data, respectively. Two dashed lines in Fig. 9 indicate the time period when the MF burst is detected. Figure 9(e) and 9(f) shows expanded dynamic spectra of LH and RH components obtained from 1540 to 1640 UT. The present MF burst event can be identified as intermittent broadband emissions covering from 1.9 to $3.1 \mathrm{MHz}$ appearing only in the LH spectra, which also show that the MF burst is generated as a L-O mode plasma wave. The MF burst occurred early in the evening while AE showed activity of $1100 \mathrm{nT}$ near $2220 \mathrm{UT}$. This event is characterized as being of long duration since it continued for several tens of minutes. LaBelle et al. (1997) reported that MF bursts usually appear in association with auroral breakup. Although no auroral images were recorded due to the strong twilight of the early evening hours, the geomagnetic data suggests that auroral activity near the observation site was relatively low when the MF burst appeared. However, it is noted that fadeout of the MF burst is clearly accompanied by the strong absorption of MF radio waves in wide frequency range. When we take into account the auroral absorption event discussed on Fig. 5, it may be inferred that auroral activity was enhanced simultaneously with the end of the MF burst event.

\subsection{Related auroral particle observation by the DMSP satellite}

In order to examine the spectra of auroral electrons related to the MF burst and the auroral roar, particle data obtained by the DMSP satellites are examined. We analyze the MF burst event on September 23 and the roar event on November 10. Figure 10(a) and 10(b) shows the electron's energy-time spectra covering the energy range of $30 \mathrm{eV}-30 \mathrm{keV}$ obtained by the DMSP F16 from 2150:00-2155:00 UT on September 23 and from 0121:300126:30 UT on November 10, respectively, when the conjugate footprint of the satellite was passing its closest point of the Husafell station. Because the DMSP F16 was located in the southern hemisphere for these two time periods, we examined the geomagnetic conjugate point of the 


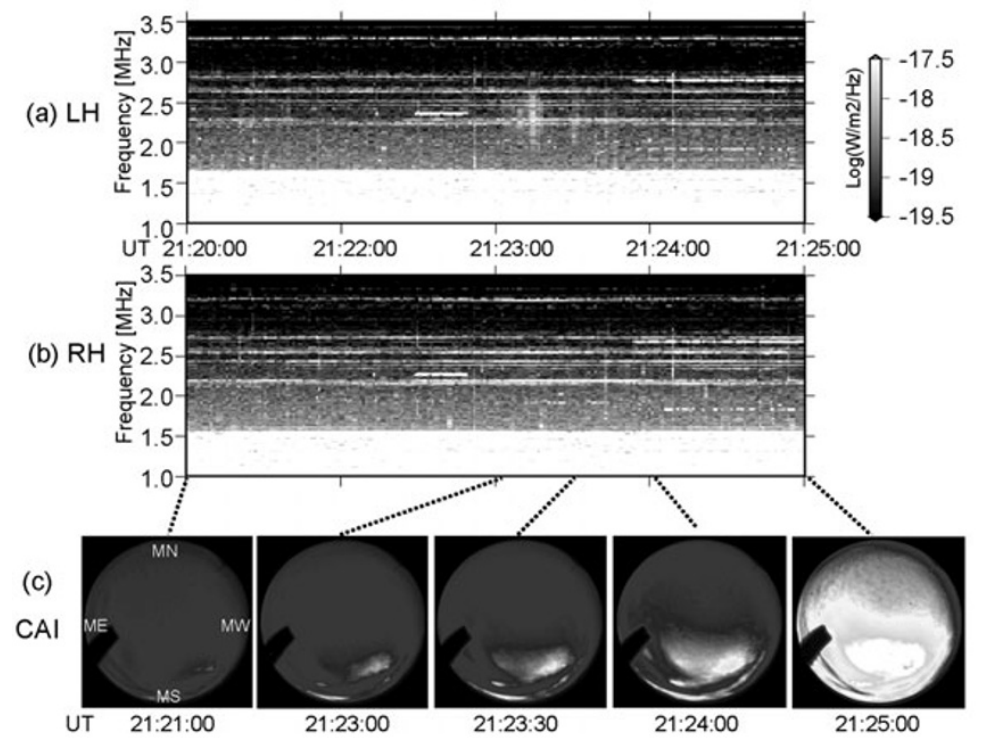

Fig. 8. Dynamic spectra of LH (panel (a)) and RH (panel (b)) components of ARS data recorded 2121-2125 UT on September 23, 2006. The MF burst appeared at 2123:10 UT only in the LH component spectrum. The panel (c) shows a series of $427.8 \mathrm{~nm}$ auroral images recorded by the Conjugate Auroral Imager (CAI) instrument located at the Husafell station.

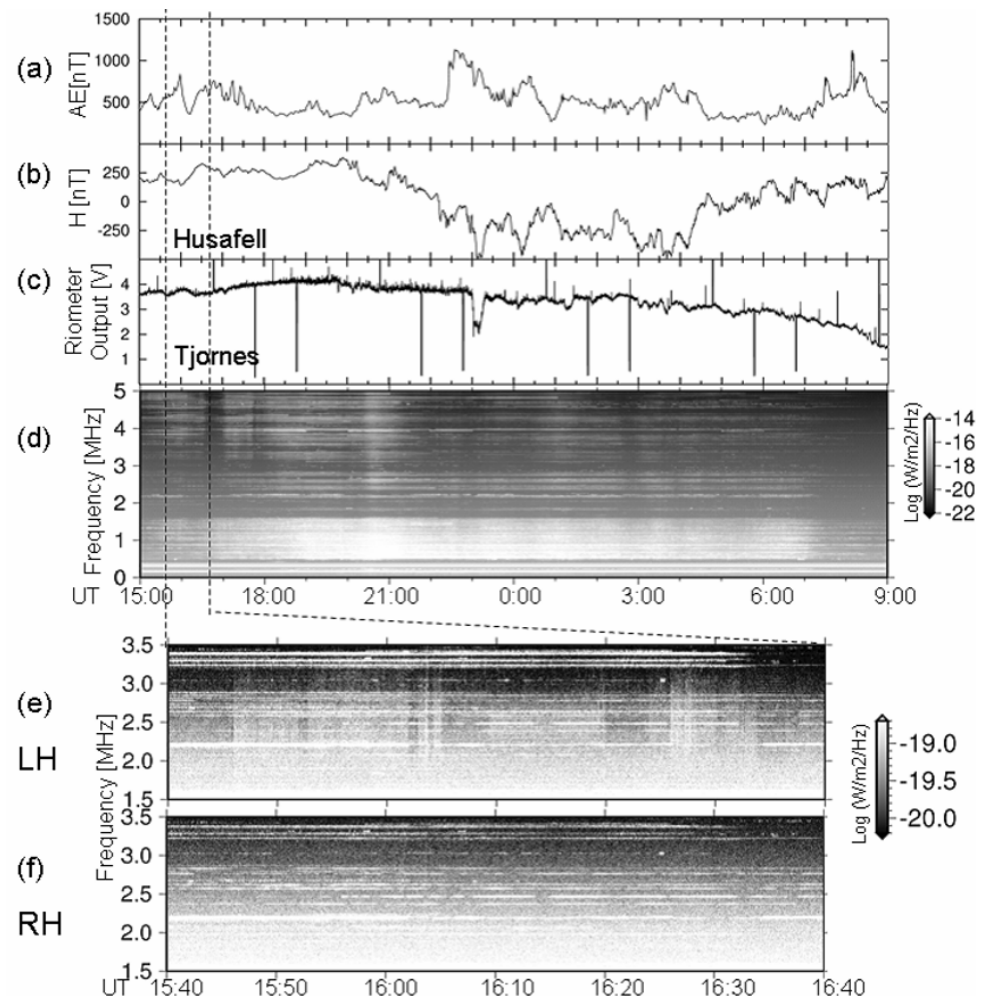

Fig. 9. The MF burst event obtained on October 28, 2006. The upper panels show (a) the AE index, (b) the $\mathrm{H}$ component of the geomagnetic field at Husafell, (c) riometer data at Tjornes, and (d) a dynamic spectrum of ARS data observed from 1500 UT on October 28 to 0900 UT on October 29, 2006. The lower panels (e) and (f) are expanded dynamic spectra showing left-handed (LH) and right-handed (RH) polarization components of the ARS data obtained 1540-1640 UT on October 28, 2006.

satellite orbits. The MF burst emission was detected at 2123:10 UT, which was about 30 min before the start time of the Fig. 10(a) plot. The auroral roar emission was observed within the period of 0115:00-0119:30 UT which was about two min before the time period of Fig. 10(b). Figure $10(\mathrm{c})$ and $10(\mathrm{~d})$ displays these satellite paths in a geomagnetic coordinate system using magnetic local time versus invariant latitude for the MF burst event and roar event, 
(a)
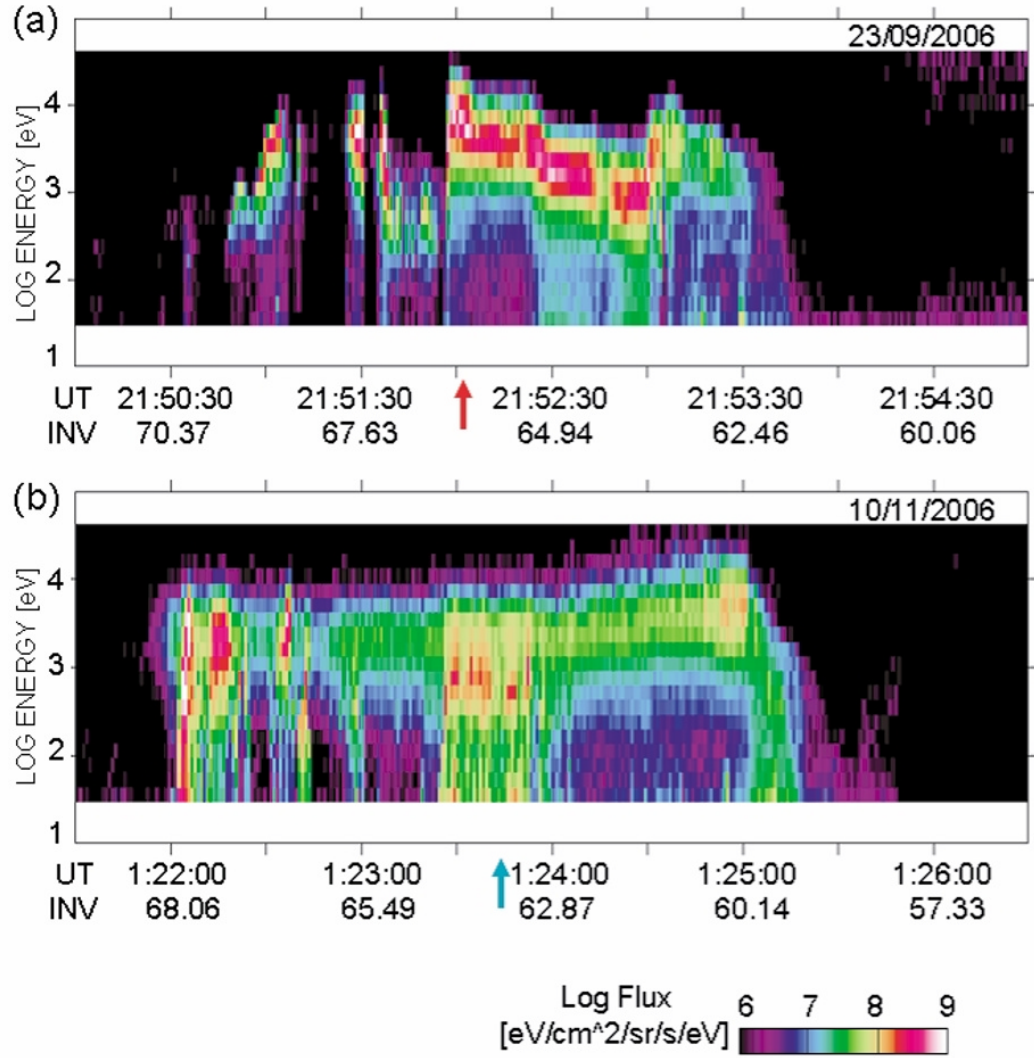
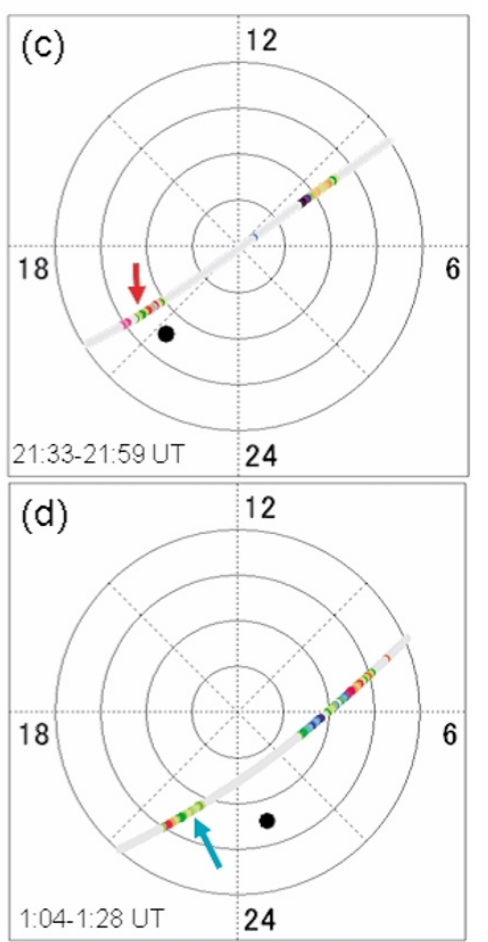

\begin{tabular}{l:l}
$1: 04-1: 28$ UT & 24 \\
\hline
\end{tabular}

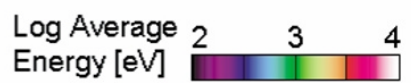

Fig. 10. Energy-time spectra of precipitating electrons covering the energy range of 30 eV-30 keV obtained by the DMSP F16 within 5 min during 2150:00-2155:00 UT on September 23 corresponding to the MF burst event (a), and 0121:30-0126:30 UT on November 10 corresponding to the auroral roar event (b), when the conjugate footprint of the satellite was passing closest to the Husafell station. (c) and (d) display electron's average energy along the satellite paths in a geomagnetic coordinate system using magnetic local time versus invariant latitude for the MF burst event and the roar event, respectively.

respectively. Each filled circle in Fig. 10(c) and 10(d) indicates the location of the Husafell station. These satellite paths are plotted by using a color code which indicates the electron's average energy within $100 \mathrm{eV}-10 \mathrm{keV}$. The weak precipitation region where the total energy flux is lower than $2 \times 10^{10} \mathrm{eV} / \mathrm{cm}^{2} / \mathrm{s} / \mathrm{sr}$ is shown in gray color.

For the case of the MF burst event, high-energy electrons more than several $\mathrm{keV}$ are dominant when we compare the average energy and peak of the total energy flux obtained near the geomagnetic latitude of the Husafell station $\left(65.3^{\circ}\right)$ (see the arrows in Fig. 10(a) and 10(c)). The result of the ground based observation given in Section 3.3 shows that the appearance of the MF burst seems to be closely related to active auroral phenomena, and it tends to disappear with the onset of the auroral breakup. These two lines of evidence obtained from the space and ground seem to be consistent each other. On the other hand, in the case of the auroral roar, a close relation can be found with the precipitation of relatively low energy electrons around the Husafell station (see the arrows in Fig. 10(b) and 10(d)). It is thus suggested that the MF bursts are generated by the electrons with an average energy of several $\mathrm{keV}$ associated with auroral breakup; on the other hand, the auroral roar emission is generated by relatively low energy electrons.

\section{Discussion and Conclusion}

In order to study the generation and propagation processes of the MF auroral radio emissions in the polar ionosphere, the ARS system was installed at the Husafell station in Iceland. Observation data were investigated in terms of ionospheric absorption and MF auroral radio emissions. To understand the occurrence and propagation character of the MF radio emissions, it is necessary to examine the nature of ionospheric absorption. Normal absorption depending on the solar zenith angle on a geomagnetically quiet day was analyzed using a simplified propagation model. The results of the data analysis clearly showed that the majority of distant man-made transmissions observed by the ARS came from the direction of the east under the strong effects of absorption and reflection in the bottomside ionosphere region near the longitude of 353E. We examined auroral absorption associated with auroral breakup as well as Pc4 range pulsating auroras. It is concluded that the ARS can detect the auroral activities indicating variations of auroral ionization occurring at distant regions from the Husafell station.

Among the observation data obtained from September to November, 2006, one auroral roar and two MF burst events were detected. This occurrence character is the same as that reported by Hughes and LaBelle (1998), that the occurrence probability is extremely low in low-latitude region of the auroral zone. It might also be related to the condition 
Auroral roar MF burst

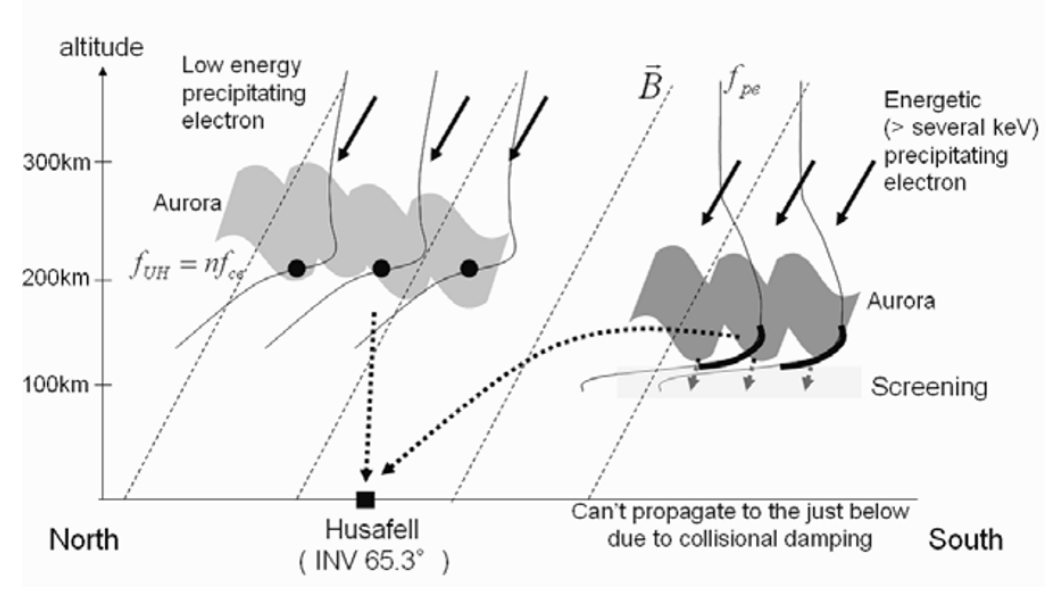

Fig. 11. Proposed generation mechanism of the auroral roar and the MF burst. The MF burst is generated in the auroral arc of the lower latitude region associated with precipitating electrons of higher energy ( $>$ several keV). It may propagate to the observation site and disappear with the beginning of strong ionization of the ionosphere. The auroral roar emission is likely to be generated as upper hybrid waves by relatively low-energy electrons over the observation point.

that during the solar minimum phase, the auroral oval tends to be contracted rather than at a solar maximum. However, these radio emissions clearly show that both $3 f_{c e}$ roar and MF bursts are LH polarized waves. Polarization character provides important information to understand the generation mechanisms in terms of the plasma instability. Among the many candidates of plasma instabilities to generate natural radio waves, the cyclotron maser instability ( $\mathrm{Wu}$ and Lee, 1979) provides the RH polarized radiation. On the other hand, the mode conversion process (Oya, 1971) from upper hybrid waves to the L-O mode radio wave likely describes the LH polarization character. One of the most probable mechanisms is that auroral roar is generated due to the mode conversion of upper hybrid waves to the LH polarized ordinary mode electromagnetic waves. The possible mechanism of the generation of the upper hybrid waves may be the loss cone plasma instability in the auroral ionosphere where upper hybrid frequency matches cyclotron harmonics (Weatherwax et al., 1995; Yoon et al., 1998; Weatherwax et al., 2002). This hypothesis has been partially verified for the $2 f_{c e}$ auroral roar by Shepherd et al. (1997). Thus, our observation result of the $3 f_{c e}$ roar supports this proposed generation mechanism. It is well known that high-temperature anisotropy of the suprathermal electrons in plasma medium results in intense plasma instability, and it is further intensified when the upper hybrid frequency matches the harmonics of the electron cyclotron frequency (Shawhan, 1979).

In the MF burst event observed on September 23, 2006, the auroral breakup occurred about $1 \mathrm{~min}$ after the termination of the MF burst. The appearance of the auroral roar observed on November 10, 2006 did not show clear correspondence with the temporal change of the geomagnetic activity. In addition, the particle observation by the DMSP satellites indicates that relatively low-energy electrons (less than $1 \mathrm{keV}$ ) are associated with the auroral roar emission. Precipitation of low-energy electrons causes ionization in a relatively high-altitude region. In other words, it does not ionize the lower altitude region of the ionosphere. For example, $0.5-\mathrm{keV}$ electrons ionize near an altitude range of $200 \mathrm{~km}$ (Ono and Hirasawa, 1992). In this case, the MF radio waves can propagate downward passing through the low-altitude regions of the ionosphere.

Compiling the observational evidence and the theoretical consideration leads to Fig. 11 which shows the occurrence of the MF burst and the auroral roar emissions. The MF burst is generated in the auroral arc of the lower latitude region associated with precipitating higher energy electrons ( $>$ several $\mathrm{keV}$ ). It can not propagate downward due to the collisional damping. MF burst waves propagating with a large horizontal component are able to arrive at the observation site on the ground, as illustrated in Fig. 11. It may propagate to the observation site and disappear with the beginning of strong ionization in the ionosphere. The auroral roar emission is likely generated as the upper hybrid wave by the relatively low-energy electrons over the observation point and propagates downward while being converted into the L-O mode electromagnetic wave. The detail verification of one-to-one correspondence between auroral radio emissions and precipitating electrons still remains for a future study; however, the present study revealed a new aspect of the physics of auroral radio emissions. In order to verify our scenario of the auroral radio emissions, simultaneous observations of auroral images and ionospheric plasma from the ground and in-situ auroral particle measurement are necessary for a future study.

Acknowledgments. We thank Dr. Gunnlaugur Björnsson and Mr. Snorri H. Johannesson for their support and operation of the ARS and the other instruments at the Husafell station in Iceland. The $\mathrm{AE}$ data were available from the WDC-C2 for Geomagnetism at Kyoto University, Japan. The auroral particle data were obtained from the URL of http://sd-www.jhuapl.edu/Aurora/. We thank Drs. D. Hardy, F. Rich, and P. Newell for their efforts. 


\section{References}

Bale, S. D., Observation of topside ionosphere MF/HF radio emission from space, Geophys. Res. Lett., 26, 667-670, 1999.

Benson, R. F. and H. K. Wong, Low-altitude ISIS observations of auroral radio emissions and their significance to the cyclotron maser instability, J. Geophys. Res., 92, 1218-1230, 1987.

Hughes, J. M. and J. LaBelle, The latitude dependence of auroral roar, $J$. Geophys. Res., 103, 14911-14916, 1998.

James, H. G., E. L. Hagg, and D. L. P. Strange, Narrowband radio noise in the topside ionosphere, AGARD Conf. Proc., AGARD-CP-138, 24-1, 1974.

Kelley, M. C., The Earth's ionosphere: plasma physics and electrodynamics, 487 pp, Academic Press, San Diego, 1989.

Kellogg, P. J. and S. J. Monson, Radio emissions from the aurora, Geophys. Res. Lett., 6, 297-300, 1979.

Kellogg, P. J. and S. J. Monson, Further studies of auroral roar, Radio Sci., 19, 551-555, 1984.

LaBelle, J. and R. A. Treumann, Auroral radio emissions, 1. Hisses, roars, and bursts, Space Sci. Rev., 101, 99-440, 2002.

LaBelle, J., A. T. Weatherwax, M. L. Trimpi, R. Brittain, R. D. Hunsucker, and J. V. Olson, The spectrum of LF/MF/HF radio noise at ground level during Substorms, Geophys. Res. Lett., 21, 2749-2752, 1994.

LaBelle, J., S. G. Shepherd, and M. L. Trimpi, Observations of auroral medium frequency bursts, J. Geophys. Res., 102, 22221-22231, 1997.

LaBelle, J., A. T. Weatherwax, M. Tantiwiwat, E. Jackson, and J. Linder, Statistical studies of auroral MF burst emissions observed at South Pole Station and at multiple sites in northern Canada, J. Geophys. Res., 110, A02305, 2005.

Ono, T. and T. Hirasawa, An apparent lifetime of auroral $630.0 \mathrm{~nm}$ (OI) Emissions, J. Geomag. Geoelectr., 44, 91-108, 1992.

Oya, H., Conversion of electrostatic plasma waves into electromagnetic waves: numerical calculation of the dispersion relation for all wavelengths, Radio Sci., 6, 1131-1141, 1971.

Oya, H., A. Morioka, and T. Obara, Leaked AKR and terrestrial hectometric radiations discovered by the plasma wave and planetary plasma sounder experiments on board the Ohzora (EXOS-C) satellite: Instrumentation and observation results of plasma wave phenomena, J. Geomag. Geoelectr., 37, 237, 1985.

Shepherd, S. G., J. LaBelle, and M. L. Trimpi, The polarization of auroral radio emissions, Geophys. Res. Lett., 24, 3161-3164, 1997.

Shepherd, S. G., J. LaBelle, C. W. Carlson, and G. Rostoker, Latitudinal dynamics of auroral roar emissions, J. Geophys. Res., 104, 1721717232, 1999.

Shawhan, S. D., Magnetospheric plasma wave research 1975-1978, Rev. Geophys. Space Phys., 17, 705-724, 1979.

Weatherwax, A. T., J. LaBelle, M. L. Trimpi, and R. Brittain, Groundbased observations of radio emissions near $2 \mathrm{f}_{\mathrm{ce}}$ and $3 \mathrm{f}_{\mathrm{ce}}$ in the auroral zone, Geophys. Res. Lett., 20, 1447-1450, 1993.

Weatherwax, A. T., J. LaBelle, and M. L. Trimpi, A new type of auroral radio emission observed at medium frequencies $(\sim 1350-3700 \mathrm{kHz})$ using ground-based receivers, Geophys. Res. Lett., 21, 2753-2756, 1994.

Weatherwax, A. T., J. LaBelle, M. L. Trimpi, R. A. Treumann, J. Minow, and C. Deehr, Statistical and case studies of radio emissions observed near $2 \mathrm{fce}$ and $3 \mathrm{fce}$ in the auroral zone, J. Geophys. Res., 100, 77457757, 1995.

Weatherwax, A. T., P. H. Yoon, and J. LaBelle, Interpreting observations of $\mathrm{MF} / \mathrm{HF}$ radio emissions: Unstable wave modes and possibilities to passively diagnose ionospheric densities, J. Geophys. Res., 107, 26-1-9, 2002.

$\mathrm{Wu}, \mathrm{C}$. S. and L. C. Lee, A theory of the terrestrial kilometric radiation, Astrophys. J., 230, 621-626, 1979.

Yoon, P. H., A. T. Weatherwax, and T. J. Rosenberg, On the generation of auroral radio emissions at harmonics of the lower ionospheric electron cyclotron frequency: X, O and $\mathrm{Z}$ mode maser calculations, J. Geophys. Res., 103, 4071-4078, 1998.

Y. Sato (e-mail: yuka@stpp1.geophys.tohoku.ac.jp), T. Ono, M. Iizima, A. Kumamoto, N. Sato, A. Kadokura, and H. Miyaoka 